Supporting Information for

\title{
Stereoselective Intramolecular 1,3 C-H Insertion in Rh(II) Carbene Reactions
}

Weifeng Shi, Bo Zhang, Jian Zhang, Binge Liu, Shiwei Zhang and Jianbo Wang*

Key Laboratory of Bioorganic Chemistry and Molecular Engineering of Ministry of Education, College of Chemistry, Peking University, Beijing 100871, China

Contents

Experimental procedure and spectra data. $\mathrm{S} 1 \sim \mathrm{S} 5$

X-ray structure of $\mathbf{2 b}$. .S5 
General information. All reactions with air- and moisture-sensitive components were performed under a nitrogen atmosphere in a flame-dried reaction flask. All solvents were distilled prior to use. $\mathrm{CH}_{2} \mathrm{Cl}_{2}$ was freshly distilled from $\mathrm{CaH}_{2}$ and THF was freshly distilled from $\mathrm{Na}$ before use. For chromatography, 200-300 mesh silica gel (Qingdao, China) was employed. ${ }^{1} \mathrm{H}$ and ${ }^{13} \mathrm{C}$ NMR spectra were recorded at 200 and $50 \mathrm{MHz}$ with a Varian Mercury 200 spectrometer or 300 and $75 \mathrm{MHz}$ with a Varian Mercury 300 spectrometer. Chemical shifts are reported by ppm using tetramethylsilane as internal standard. Mass spectra were obtained on a VG ZAB-HS mass spectrometer.

\section{General procedure for nucleophilic substitution of 1a e with sodium $p$-toluenesulfinate.}

In a dry two-necked flask were added sodium $p$-toluenesulfinate $(2 \mathrm{mmol})$ and DMF (30 $\mathrm{mL})$. The mixture was stirred until it turned to be a homogenous solution. $\beta$-Acetoxy $\alpha$-diazo carbonyl compound $\mathbf{1 a} \sim \mathbf{e}(1 \mathrm{mmol})$ was added to the solution and stirring was continued for 7 days until all the starting material disappeared as judged by TLC check. DMF was removed under reduced pressure and distilled water was then added to the crude residue. The mixture was extracted with ethyl ether $(3 \times 15 \mathrm{~mL})$. The combined ethereal solution was washed with saturated aqueous $\mathrm{NaCl}$ and then dried with anhydrous $\mathrm{MgSO}_{4}$. Removal of the solvent gave a crude product, which was purified by silica gel column.

Ethyl 2-Diazo-3-(p-tolylsulfonyl)-pentanote (2a). TLC $R_{f}=0.21$ (petroleum ether : acetone $=10: 1)$; IR 2981, 2099, 1699, 1286, $1145 \mathrm{~cm}^{-1} ;{ }^{1} \mathrm{H}$ NMR $\left(300 \mathrm{MHz}, \mathrm{CDCl}_{3}\right)$ $\delta$ 1.05-1.10 (m, 6H), 1.65-1.79 (m, 1H), 2.39-2.46 (m, 1H), $2.43(\mathrm{~s}, 3 \mathrm{H}), 3.80-3.99(\mathrm{br}, 3 \mathrm{H})$, $7.33(\mathrm{~d}, J=8.1 \mathrm{~Hz}, 2 \mathrm{H}), 7.74(\mathrm{~d}, J=8.4 \mathrm{~Hz}, 2 \mathrm{H}) ;{ }^{13} \mathrm{C} \mathrm{NMR}\left(50 \mathrm{MHz}, \mathrm{CDCl}_{3}\right) \delta 11.07,14.18$, 17.48, 21.59, 61.09, 61.28, 128.63, 129.56, 134.25, 144.64; MS m/z (EI) 310 (M $\left.\mathrm{M}^{+}, 0.55\right), 282$ $\left[(\mathrm{M}-28)^{+}, 0.36\right], 218$ (3), 172 (4), 155 (24), 127 (72), 91 (95), 81 (26), 65 (33), 55 (43), 29 (100). HMRS calcd for 310.0987. Found: 310.0997.

Ethyl 2-Diazo-3-(p-tolylsulfonyl)-hexanoate (2b). TLC $R_{f}=0.21$ (petroleum ether : acetone $=10: 1)$; IR 2964, 2099, 1698, 1321, $1142 \mathrm{~cm}^{-1} ;{ }^{1} \mathrm{H}$ NMR $\left(200 \mathrm{MHz}, \mathrm{CDCl}_{3}\right)$

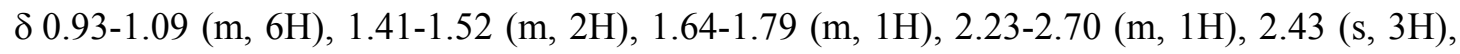
3.86-3.98 (m, 3H), $7.33(\mathrm{~d}, J=8.2 \mathrm{~Hz}, 2 \mathrm{H}), 7.76(\mathrm{~d}, J=8.2 \mathrm{~Hz}, 2 \mathrm{H}) ;{ }^{13} \mathrm{C}$ NMR $(50 \mathrm{MHz}$, $\left.\mathrm{CDCl}_{3}\right) \delta 13.43,14.15,19.74,21.55,25.47,59.32,61.22,128.72,129.61,134.43,144.96$, 154.96; MS m/z (EI) 324 (M+, 0.09), 296 [(M-28) $\left.{ }^{+}, 0.02\right], 251$ (9), 169 (55), 139 (98), 113 (100), 91 (95), 65 (64), 55 (48). Anal. calcd for $\mathrm{C}_{15} \mathrm{H}_{20} \mathrm{~N}_{2} \mathrm{O}_{4} \mathrm{~S}$ : C, 55.54; H, 6.21; N, 8.64. Found: C, 55.56; H, 6.26; N, 8.65.

3-Diazo-4-( $\boldsymbol{p}$-tolylsulfonyl)-hexan-2-one (2c). TLC $R_{f}=0.29$ (petroleum ether : acetone $=$ 5 : 1); IR 2917, 2086, 1649, 1315, $1145 \mathrm{~cm}^{-1} ;{ }^{1} \mathrm{H}$ NMR $\left(200 \mathrm{MHz}, \mathrm{CDCl}_{3}\right) \delta 1.02$ (t, $J=7.2 \mathrm{~Hz}$, $3 \mathrm{H}), 1.67-1.77$ (m, 1H), 2.06 (s, 3H), 2.29-2.47 (m, 1H), 2.43 (s, 3H), 4.07 (br., d, $J=11 \mathrm{~Hz}$, $1 \mathrm{H}), 7.33(\mathrm{~d}, J=7.6 \mathrm{~Hz}, 2 \mathrm{H}), 7.74(\mathrm{~d}, J=9.6 \mathrm{~Hz}, 2 \mathrm{H}) ;{ }^{13} \mathrm{C} \mathrm{NMR}\left(50 \mathrm{MHz}, \mathrm{CDCl}_{3}\right) \delta 10.91$, $17.25,21.59,24.66,59.02,128.36,129.62,134.21,145.11,188.19$. MS $m / z$ (EI) $280\left(\mathrm{M}^{+}\right.$, 0.36), 252 (0.26), 150 (15), 139 (39), 119 (22), 97 (87), 65 (33), 43(100), 41 (32). Anal. calcd for $\mathrm{C}_{13} \mathrm{H}_{16} \mathrm{~N}_{2} \mathrm{O}_{3} \mathrm{~S}: \mathrm{C}, 55.70 ; \mathrm{H}, 5.75 ; \mathrm{N}, 9.99$. Found: $\mathrm{C}, 55.74 ; \mathrm{H}, 5.79 ; \mathrm{N}, 9.86$. 
1-Diazo-2-(p-tolylsulfonyl)butyl phenyl ketone (2d). TLC $R_{f}=0.39$ (petroleum ether : acetone $=5: 1)$; IR 2928, 2083, 1621, 1292, $1148 \mathrm{~cm}^{-1} ;{ }^{1} \mathrm{H}$ NMR $\left(300 \mathrm{MHz}, \mathrm{CDCl}_{3}\right) \delta 1.13$ (t, $J=7.5 \mathrm{~Hz}, 3 \mathrm{H}), 1.74-1.90$ (m, 1H), 2.43-2.52 (m, 1H), 2.40 (s, 3H), 4.30 (br, 1H), 7.27-7.51 $(\mathrm{m}, 7 \mathrm{H}), 7.80-7.86(\mathrm{~m}, 2 \mathrm{H}) ;{ }^{13} \mathrm{C} \mathrm{NMR}\left(75 \mathrm{MHz}, \mathrm{CDCl}_{3}\right) \delta 11.02,17.19,21.60,59.80,126.74$, 129.04, 129.73, 129.91, 131.82, 134.35, 136.20, 145.17. MS m/z (EI) 314 [(M-28) $\left.{ }^{+}, 1.00\right], 176$ (55), 159 (37), 139 (41), 131 (97), 115 (44), 105 (100), 91 (87), 77 (83). Anal. calcd for $\mathrm{C}_{18} \mathrm{H}_{18} \mathrm{~N}_{2} \mathrm{O}_{3} \mathrm{~S}: \mathrm{C}, 63.14 ; \mathrm{H}, 5.30 ; \mathrm{N}, 8.18$. Found: $\mathrm{C}, 62.96 ; \mathrm{H}, 5.36 ; \mathrm{N}, 7.84$.

Ethyl 2-Diazo-3-(p-tolylsulfonyl)-heptanoate (2e). TLC $R_{f}=0.38$ (petroleum ether : acetone $=5: 1)$; IR 2959, 2097, 1698, 1322, $1146 \mathrm{~cm}^{-1} ;{ }^{1} \mathrm{H}$ NMR $\left(200 \mathrm{MHz}, \mathrm{CDCl}_{3}\right) \delta 0.91$ (t, $J=7.2 \mathrm{~Hz}$, $3 \mathrm{H}), 1.06$ (t, $J=6.3 \mathrm{~Hz}, 3 \mathrm{H}), 1.26-1.80(\mathrm{br}, 5 \mathrm{H}), 2.23-2.33$ (br, $1 \mathrm{H}), 2.43$ (s, 3H), 3.84-3.99 (br, $4 \mathrm{H}), 7.33(\mathrm{~d}, J=7.8 \mathrm{~Hz}, 2 \mathrm{H}), 7.73-7.84(\mathrm{~m}, 2 \mathrm{H}) ;{ }^{13} \mathrm{C} \mathrm{NMR}\left(50 \mathrm{MHz}, \mathrm{CDCl}_{3}\right) \delta 13.56,14.05$, 21.47, 21.92, 23.10, 28.40, 59.49, 61.18, 128.77, 129.65, 134.45, 145.04, 164.92; MS m/z (EI) 338 $\left(\mathrm{M}^{+}, 0.20\right), 310$ (0.15), 127 (77), 109 (50), 91 (66), 81 (92), 55 (49), 29 (100). Anal. Calcd for $\mathrm{C}_{16} \mathrm{H}_{22} \mathrm{~N}_{2} \mathrm{O}_{4} \mathrm{~S}: \mathrm{C}, 56.78 ; \mathrm{H}, 6.55 ; \mathrm{N}, 8.28$. Found: C, 56.76; H, 6.58; N, 8.28.

Ethyl 2-Diazo-3-( $\boldsymbol{p}$-tolylsulfonyl)-nonanoate (2f). TLC $R_{f}=0.59$ (petroleum ether : acetone $=5: 1$ ); IR 2929, 2098, 1698, 1324, $1141 \mathrm{~cm}^{-1} ;{ }^{1} \mathrm{H} \mathrm{NMR}\left(400 \mathrm{MHz}, \mathrm{CDCl}_{3}\right) \delta 0.87$ (t, 3H, $J=$ $6.7 \mathrm{~Hz}), 1.07$ (br, 3H), 1.26-1.43 (m, 8H), 1.61-1.73 (m, 1H), $2.27(\mathrm{br}, 1 \mathrm{H}), 2.43(\mathrm{~s}, 3 \mathrm{H})$, $3.86-3.98(\mathrm{~m}, \mathrm{br}, 3 \mathrm{H}), 7.33(\mathrm{~d}, 2 \mathrm{H}, J=8.1 \mathrm{~Hz}), 7.76(\mathrm{~d}, 2 \mathrm{H}, J=8.2 \mathrm{~Hz}) ;{ }^{13} \mathrm{C}$ NMR $(100 \mathrm{MHz}$, $\left.\mathrm{CDCl}_{3}\right) \delta 13.9,14.2,21.6,22.4,23.5,26.4,28.5,31.3,59.6,61.2,128.8,129.6,134.5,145.0$; MS m/z (EI) 366 (M+, 0.38), 338 (0.69), 293 (3), 211 (10) 183 (62), 155 (51), 139 (52), 109 (100), 91 (81), 67 (39), 55 (47), 29 (99). Anal. Calcd for $\mathrm{C}_{18} \mathrm{H}_{26} \mathrm{SO}_{4} \mathrm{~N}_{2}: \mathrm{C}, 58.99 ; \mathrm{H}, 7.15 ; \mathrm{N}$, 7.64. Found: C, 58.96; H, 7.10; N, 7.51.

$\beta$-Tosyl $\alpha$-diazo carbonyl compounds $7 \mathbf{a}$ and $7 \mathbf{b}$ were prepared similarly from the corresponding $\beta$-hydroxy $\alpha$-diazo carbonyl compounds.

Ethyl 3-Cyclopentyl-2-diazo-3-(p-tolylsulfonyl)propanoate (7a). TLC $R_{f}=0.34$ (petroleum ether : acetone $=10: 1)$; IR 2960, 2096, 1697, 1303, $1143 \mathrm{~cm}^{-1} ;{ }^{1} \mathrm{H}$ NMR $(200 \mathrm{MHz}$, $\left.\mathrm{CDCl}_{3}\right) \delta 1.03(\mathrm{t}, J=7.2 \mathrm{~Hz}, 3 \mathrm{H}), 1.26-1.44(\mathrm{~m}, 1 \mathrm{H}), 1.65-1.74(\mathrm{~m}, 5 \mathrm{H}), 1.81-1.95(\mathrm{~m}, 1 \mathrm{H})$, 2.17-2.26 (m, 1H), $2.42(\mathrm{~s}, 3 \mathrm{H}), 2.52-2.56(\mathrm{~m}, 1 \mathrm{H}), 3.80(\mathrm{~d}, J=10 \mathrm{~Hz}, 1 \mathrm{H}), 3.82-3.90(\mathrm{~m}$, $2 \mathrm{H}), 7.31(\mathrm{~d}, J=8.2 \mathrm{~Hz}, 2 \mathrm{H}), 7.76(\mathrm{~d}, J=8.2 \mathrm{~Hz}, 2 \mathrm{H}) ;{ }^{13} \mathrm{C} \mathrm{NMR}\left(75 \mathrm{MHz}, \mathrm{CDCl}_{3}\right) \delta 14.04$, $21.41,23.94,25.32,31.03,31.53,36.85,61.04,63.74,128.27,129.36,135.26,144.67 . \mathrm{MS}$ m/z (EI) $350\left(\mathrm{M}^{+}, 0.24\right), 195$ (13), 167 (15), 139 (100), 121 (67), 91 (60), 65 (26), 29 (45). Anal. calcd for $\mathrm{C}_{17} \mathrm{H}_{22} \mathrm{~N}_{2} \mathrm{O}_{4} \mathrm{~S}: \mathrm{C}, 58.27 ; \mathrm{H}, 6.33 ; \mathrm{N}, 7.99$. Found: C, 58.25; H, 6.38; N, 7.99.

Ethyl 3-Cyclohexyl-2-diazo-3-(p-tolylsulfonyl)propanoate (7b). TLC $R_{f}=0.39$ (petroleum ether : acetone $=10: 1)$; IR 2930, 2097, 1697, 1318, $1142 \mathrm{~cm}^{-1} ;{ }^{1} \mathrm{H}$ NMR $(300 \mathrm{MHz}$, $\left.\mathrm{CDCl}_{3}\right) \delta 1.03-1.42(\mathrm{~m}, 9 \mathrm{H}), 1.63-2.04(\mathrm{~m}, 5 \mathrm{H}), 2.42(\mathrm{~s}, 3 \mathrm{H}), 3.75-3.93(\mathrm{~m}, 3 \mathrm{H}), 7.32(\mathrm{~d}, J=$ $8.1 \mathrm{~Hz}, 2 \mathrm{H}), 7.76(\mathrm{~d}, J=8.1 \mathrm{~Hz}, 2 \mathrm{H}) ;{ }^{13} \mathrm{C} \mathrm{NMR}\left(75 \mathrm{MHz}, \mathrm{CDCl}_{3}\right) \delta 14.22,21.57,25.82$, $25.92,25.96,29.89,31.88,36.90,61.27,63.97,128.11,129.57,135.85,144.75$. MS $m / z$ (EI) 181 (29), 153 (61), 135 (100), 107 (76), 91 (62), 79 (48), 67 (41). Anal. calcd for $\mathrm{C}_{18} \mathrm{H}_{24} \mathrm{~N}_{2} \mathrm{O}_{4} \mathrm{~S}: \mathrm{C}$, 59.32; H, 6.64; N, 7.69. Found: C, 59.37; H, 6.60; N, 7.64. 


\section{General procedure for $\mathrm{Rh}_{2}(\mathrm{OAc})_{4}$-catalyzed reaction of $2 \mathrm{a} \sim \mathrm{e}$ and $7 \mathrm{a}, \mathrm{b}$.}

Freshly distilled $\mathrm{CH}_{2} \mathrm{Cl}_{2}$ or benzene $(30 \mathrm{~mL})$ was treated by bubbling with $\mathrm{N}_{2}$ gas for 10 min and then sealed with rubber stopper. Under a stream of $\mathrm{N}_{2}$ gas, $\mathrm{Rh}_{2}(\mathrm{OAc})_{4}(5 \% \mathrm{mmol})$ was added to a three-necked round-bottom flask, which was equipped with a drop funnel. To the flask was added the $\mathrm{N}_{2}$-treated $\mathrm{CH}_{2} \mathrm{Cl}_{2}$ or benzene. The diazo compound ( $1 \mathrm{mmol}$ ) was dissolved in the above $\mathrm{N}_{2}$-treated $\mathrm{CH}_{2} \mathrm{Cl}_{2}$ or benzene and added to the flask through drop funnel. The system was kept under $\mathrm{N}_{2}$ atmosphere with a balloon. The stirring was continued for $12 \mathrm{~h}$, during which the $\mathrm{N}_{2}$ balloon is changed several times in order to strictly excluding oxygen. After completion of the reaction, solvent was removed under reduced pressure and the crude residue was separated with silica gel column (petroleum ether : acetone $=10: 1$ ).

Similar procedure was followed when the reaction was carried out under reflux.

$r$-1, trans-2, trans-3, Ethyl 1-Methyl-3-(p-tolylsulfonyl)cyclopropylformate (3a). TLC $R_{f}$ $=0.09$ (petroleum ether : acetone $=10: 1)$; IR 2980, 1735, 1317, $1151 \mathrm{~cm}^{-1} ;{ }^{1} \mathrm{H}$ NMR $(400$ $\left.\mathrm{MHz}, \mathrm{CDCl}_{3}\right) \delta 1.20(\mathrm{~d}, J=6.1 \mathrm{~Hz}, 3 \mathrm{H}), 1.27(\mathrm{t}, J=7.2 \mathrm{~Hz}, 3 \mathrm{H}), 1.99(\mathrm{dd}, J=9.0,7.1 \mathrm{~Hz}$, $1 \mathrm{H}), 2.39-2.43(\mathrm{~m}, 1 \mathrm{H}), 2.44(\mathrm{~s}, 3 \mathrm{H}), 2.56(\mathrm{dd}, J=9.0,6.1 \mathrm{~Hz}, 1 \mathrm{H}), 4.13-4.21(\mathrm{~m}, 2 \mathrm{H}), 7.34$ $(\mathrm{d}, J=8.1 \mathrm{~Hz}, 2 \mathrm{H}), 7.78(\mathrm{~d}, J=8.3 \mathrm{~Hz}, 2 \mathrm{H}) ;{ }^{13} \mathrm{C} \mathrm{NMR}\left(50 \mathrm{MHz}, \mathrm{CDCl}_{3}\right) \delta 13.75,16.10,19.22$, 21.40, 30.64, 46.73, 61.39, 127.55, 129.65, 137.71, 144.44, 167.08; MS m/z (EI) $282\left(\mathrm{M}^{+}, 2\right)$, 237 (11), 209 (5), 155 (5), 139 (15), 127 (65), 99 (100), 91 (31), 81 (12), 71 (13), 65 (15), 53 (15), 43 (37), 39 (16), 29 (23), 27 (18) . Anal. calcd for $\mathrm{C}_{14} \mathrm{H}_{18} \mathrm{O}_{4} \mathrm{~S}: \mathrm{C}, 59.55 ; \mathrm{H}, 6.43$. Found: C, $59.37 ; \mathrm{H}, 6.55$.

$r$-1, trans-2, trans-3, Ethyl 1-Ethyl-3-(p-tolylsulfonyl)cyclopropylformate (3b). TLC $R_{f}$ $=0.11$ (petroleum ether : acetone $=10: 1) ;$ IR 2966, 1737, 1324, $1144 \mathrm{~cm}^{-1} ;{ }^{1} \mathrm{H}$ NMR $(200$ $\left.\mathrm{MHz}, \mathrm{CDCl}_{3}\right) \delta 0.91(\mathrm{t}, J=7.2 \mathrm{~Hz}, 3 \mathrm{H}), 1.33(\mathrm{t}, J=7.2 \mathrm{~Hz}, 3 \mathrm{H}), 1.39-1.64(\mathrm{~m}, 2 \mathrm{H}), 2.03(\mathrm{dd}$, $J=9.2,7.0 \mathrm{~Hz}, 1 \mathrm{H}), 2.28-2.41(\mathrm{~m}, 1 \mathrm{H}), 2.45(\mathrm{~s}, 3 \mathrm{H}), 2.55(\mathrm{dd}, J=9.2,6.2 \mathrm{~Hz}, 1 \mathrm{H}), 4.19$ (q, $J$ $=7.2 \mathrm{~Hz}, 2 \mathrm{H}), 7.34(\mathrm{~d}, J=8.2 \mathrm{~Hz}, 2 \mathrm{H}), 7.79(\mathrm{~d}, J=8.2 \mathrm{~Hz}, 2 \mathrm{H}) ;{ }^{13} \mathrm{C}$ NMR $\left(50 \mathrm{MHz}, \mathrm{CDCl}_{3}\right)$ $\delta 12.44,13.94,21.59,24.39,26.31,29.75,45.89,61.56,127.72,129.64,137.61,144.45$, 167.15; MS m/z (EI) 296 (M+ , 0.70), 251 (14), 223 (7), 141 (99), 139 (18), 113 (100), 95 (62), 91 (33), 67 (25), 41 (23), 29 (27). Anal. calcd for $\mathrm{C}_{15} \mathrm{H}_{20} \mathrm{O}_{4} \mathrm{~S}$ : C, 60.79; H, 6.80. Found: C, $60.34 ; \mathrm{H}, 6.89$.

$r$-1, trans-2, trans-3, 1-Methyl-3-(p-tolylsulfonyl)cyclopropyl Methyl Ketone (3c). TLC $R_{f}=0.16$ (petroleum ether : acetone $=5: 1$ ); IR 2965, 1713, 1303, $1148 \mathrm{~cm}^{-1} ;{ }^{1} \mathrm{H}$ NMR $(300$ $\left.\mathrm{MHz}, \mathrm{CDCl}_{3}\right) \delta 1.14(\mathrm{~d}, J=6.0 \mathrm{~Hz}, 3 \mathrm{H}), 2.15(\mathrm{dd}, J=9.3,7.1 \mathrm{~Hz}, 1 \mathrm{H}), 2.29(\mathrm{~s}, 3 \mathrm{H})$, 2.35-2.38 (m, 1H), 2.67 (dd, $J=9.3,6.0 \mathrm{~Hz}, 1 \mathrm{H}), 7.37$ (d, $J=8.4 \mathrm{~Hz}, 2 \mathrm{H}), 7.76$ (d, $J=8.4 \mathrm{~Hz}$, $2 \mathrm{H}) ;{ }^{13} \mathrm{C} \mathrm{NMR}\left(50 \mathrm{MHz}, \mathrm{CDCl}_{3}\right) \delta 16.01,19.48,21.42,30.89,37.95,48.43,127.37,129.65$, 137.59, 144.43, 200.63; MS m/z (EI) 252 (M+, 2), 210 (3), 155 (4), 97 (100), 91 (30), 43 (99), 41 (28), 27 (12). Anal. calcd for $\mathrm{C}_{13} \mathrm{H}_{16} \mathrm{O}_{3} \mathrm{~S}: \mathrm{C}, 61.88 ; \mathrm{H}, 6.39$. Found: C, 61.72; H, 6.46.

r-1, trans-2, trans-3, 1-Methyl-3-(p-tolylsulfonyl)cyclopropyl Phenyl Ketone (3d). TLC $R_{f}=0.24$ (petroleum ether : acetone $\left.=5: 1\right)$; IR 3031, 1678, 1312, $1151 \mathrm{~cm}^{-1} ;{ }^{1} \mathrm{H}$ NMR $(300$ $\left.\mathrm{MHz}, \mathrm{CDCl}_{3}\right) \delta 1.27(\mathrm{~d}, J=5.7 \mathrm{~Hz}, 3 \mathrm{H}), 2.33(\mathrm{~s}, 3 \mathrm{H}), 2.65-2.69(\mathrm{~m}, 2 \mathrm{H}), 2.87-2.92(\mathrm{~m}, 1 \mathrm{H})$, 
$7.11(\mathrm{~d}, J=8.4 \mathrm{~Hz}, 2 \mathrm{H}), 7.32-7.37(\mathrm{~m}, 2 \mathrm{H}), 7.49-7.55(\mathrm{~m}, 3 \mathrm{H}), 7.86(\mathrm{~d}, J=8.4 \mathrm{~Hz}, 2 \mathrm{H}),{ }^{13} \mathrm{C}$ NMR (75 MHz, $\left.\mathrm{CDCl}_{3}\right) \delta 16.43,18.52,21.45,34.78,49.01,127.48,128.27,128.40,129.45$, 133.02, 136.95, 137.39, 144.17, 191.28. MS $m / z$ (EI) 314 (M+ 0.3), 250 (14), 207 (4), 159 (100), 144 (11), 131 (24), 115 (10), 105 (81), 91 (37), 77 (55), 65 (10), 43 (70). Anal. calcd for $\mathrm{C}_{18} \mathrm{H}_{18} \mathrm{O}_{3} \mathrm{~S}$ : C, 68.76; $\mathrm{H}$, 5.77. Found: $\mathrm{C}, 68.58 ; \mathrm{H}, 5.83$.

cis-1-(p-tolylsulfonyl)-2-carboethoxyspiro[2.4]heptane (8a). TLC $R_{f}=0.15$ (petroleum ether : acetone $=10: 1)$; IR 2955, 1737, 1321, $1146 \mathrm{~cm}^{-1} ;{ }^{1} \mathrm{H}$ NMR $\left(200 \mathrm{MHz}, \mathrm{CDCl}_{3}\right) \delta 1.32$ (t, $J=7.2 \mathrm{~Hz}, 3 \mathrm{H}), 1.63-1.88(\mathrm{~m}, 7 \mathrm{H}), 2.16(\mathrm{~d}, J=9.2 \mathrm{~Hz}, 1 \mathrm{H}), 2.25-2.39(\mathrm{~m}, 1 \mathrm{H}), 2.44(\mathrm{~s}$, $3 \mathrm{H}), 2.65(\mathrm{~d}, J=9.2 \mathrm{~Hz}, 1 \mathrm{H}), 4.16-4.32(\mathrm{~m}, 2 \mathrm{H}), 7.34(\mathrm{~d}, J=8.2 \mathrm{~Hz}, 2 \mathrm{H}), 7.86(\mathrm{~d}, J=8.2 \mathrm{~Hz}$, $2 \mathrm{H}) ;{ }^{13} \mathrm{C}$ NMR $\left(50 \mathrm{MHz}, \mathrm{CDCl}_{3}\right) \delta 13.96,21.55,24.19,25.87,26.06,32.09,36.10,38.21$, 47.93, 61.11, 127.45, 129.59, 138.83, 144.22, 166.76. MS m/z (EI) $277\left[(\mathrm{M}-45)^{+}, 3\right], 249$ (6), 209 (2), 167 (93), 139 (68), 121 (100), 91 (39), 77 (21), 55 (16), 41 (14), 39 (11), 27 (7). Anal. calcd for $\mathrm{C}_{17} \mathrm{H}_{22} \mathrm{O}_{4} \mathrm{~S}$ : C, 63.33; H, 6.88. Found: C, 63.30; H, 6.78.

cis-1-(p-tolylsulfonyl)-2-carboethoxyspiro[2,4]octane (8b). TLC $R_{f}=0.19$ (petroleum ether : acetone $=10: 1)$; IR 2931, 1737, 1319, $1147 \mathrm{~cm}^{-1} ;{ }^{1} \mathrm{H}$ NMR (300 MHz, $\left.\mathrm{CDCl}_{3}\right) \delta 1.23-1.39(\mathrm{~m}, 4 \mathrm{H}), 1.54-1.76(\mathrm{~m}, 7 \mathrm{H}), 1.97-2.37(\mathrm{~m}, 4 \mathrm{H}), 2.39-2.45(\mathrm{~m}, 3 \mathrm{H})$, 4.21-4.30 (m, 2H), $7.36(\mathrm{~d}, J=7.8 \mathrm{~Hz}, 2 \mathrm{H}), 7.87(\mathrm{~d}, J=8.4 \mathrm{~Hz}, 2 \mathrm{H}) ;{ }^{13} \mathrm{C}$ NMR $(75 \mathrm{MHz}$, $\left.\mathrm{CDCl}_{3}\right) \delta 13.99,21.57,25.22,25.69,33.26,33.67,38.87,48.89,61.15,127.55,129.60$, 139.15, 144.21, 166.77; MS m/z (EI) 291 [(M-45) $\left.{ }^{+}, 3\right], 263$ (5), 181 (94), 153 (15), 135 (100), 117 (14), 107 (34), 91 (42), 79 (39), 67 (26), 41 (18), 29 (29). Anal. calcd for $\mathrm{C}_{18} \mathrm{H}_{24} \mathrm{O}_{4} \mathrm{~S}: \mathrm{C}$, 64.26; H, 7.19. Found: C, 64.25; H, 7.21.

$\underline{X-r a y ~ s t r u c t u r e ~ o f ~} \mathbf{2 b}$

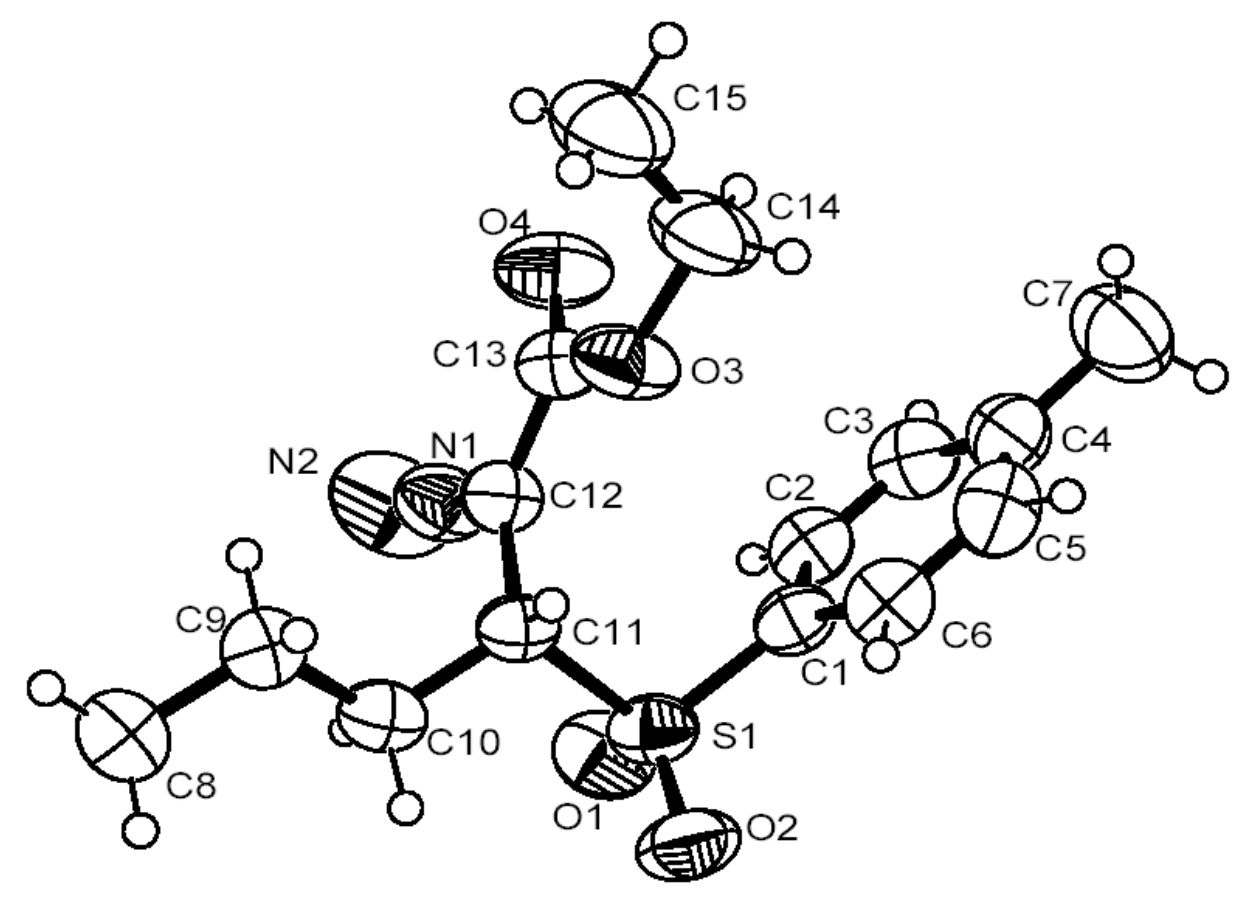

